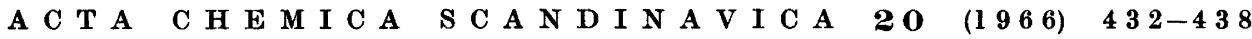

\title{
Preparation and Mechanism of Formation of the Clay-Kinnear-Perren Complex
}

\author{
GÖSTALINDNER and PER OLOF GRANBOM \\ Research Institute of National Defence, Dept. 1, Sundbyberg 4, Sweden
}

\begin{abstract}
The reaction sequence of formation of the Clay-Kinnear-Perren complex has been elucidated with thermographic and analytical methods. The reaction is supposed to start with the formation of an instable complex $\mathrm{PCl}_{2}+\mathrm{AlCl}_{4}^{-}$to which alkyl chloride is added.
\end{abstract}

In principle several ways of reaction are available for the preparation of Lalkylated phosphoryl compounds. The most important methods are reviewed by Kosolapoff, ${ }^{1}$ Crofts, ${ }^{2}$ and Frank. ${ }^{3}$ To complete the list, new ways presented by Jungerman, ${ }^{4}$ Maier, ${ }^{5}$ and Angstadt $^{6}$ have to be mentioned. Jungerman describes the addition of phosphorus trichloride to an olefin in the presence of aluminium chloride. The two latter authors have studied the alkylation of red and white phosphorus, respectively, with alkyl chloride either at high temperature ${ }^{5}$ or in the presence of aluminium chloride. 6

In preparative laboratory work, however, obstacles are met in establishing the carbon phosphorus bond, such as evolution of explosive and poisonous phosphines as a side reaction of the Arbuzov re-arrangement of trimethyl phosphite to dimethoxy methyl phosphineoxide.

The reaction sequence first suggested by Clay ${ }^{7}$ and Kinnear and Perren ${ }^{8}$

$$
\mathrm{RCl}+\mathrm{PCl}_{3}+\mathrm{AlCl}_{3} \longrightarrow \mathrm{RPCl}_{3}+\mathrm{AlCl}_{4} \stackrel{-\mathrm{H}_{2} \mathrm{O}}{\longrightarrow} \mathrm{RP}(\mathrm{O}) \mathrm{Cl}_{2}
$$

offers advantages owing to high reaction velocity, good yield, and reduced number of reaction steps. However, the total yield is strongly dependent on the reaction conditions in each step of the procedure. It was therefore felt that a closer study of the influence of reaction conditions on the formation and hydrolysis of the alkyl trichloro phosphonium teterachloro aluminate complex would be of interest.

To elucidate the reaction sequences when a mixture of alkyl chloride, phosphorus trichloride, and aluminium trichloride finally forms alkyl trichloro phosphonium tetrachloro aluminate, attempts have been made with thermographic analysis where particle size of aluminium trichloride and temperature 
were varied. Methyl chloride and ethyl chloride were chosen as alkylating agents. With ethyl chloride the consumption of phosphorus trichloride was also determined in parallel experiments by means of a phosphate analysis which excludes alkylated phosphates.

\section{EXPERIMENTAL}

Chemicals. Reagents of technical grade were used. The phosphorus trichloride was purified by distillation over phosphorus pentoxide and aluminium trichloride. Both methyl and ethyl chloride were of high purity. Special purification was not necessary. For the temperature registration experiments, the aluminium trichloride (sublimed) with about $5 \%$ oxide content was used without re-sublimation because of its convenient particle size distribution. For experiments involving chemical analysis, the aluminium trichloride was first re-sublimed.

Apparatus for thermographic analysis. The synthesis was performed in an autoclave (Fig. 1) constructed of stainless steel with a volume of $100 \mathrm{ml}$ and able to withstand a pressure of $35 \mathrm{~atm}$. It was provided with a stirrer and a teflon screw in the bottom where the thermocouple (Cu/constantan) was inserted. The junction of the thermo-couple was

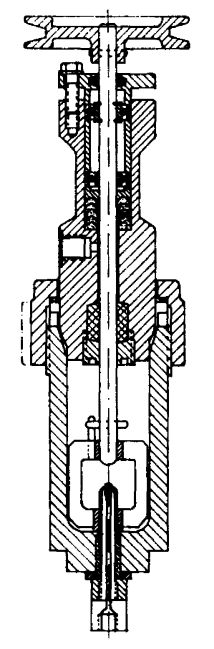

Fig. 1. Autoclave with stirrer.

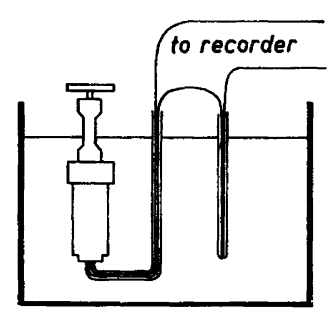

Fig. 2. The apparatus arrangement.

in contact with the reagents via a small silver plug. During the temperature registration, the autoclave was immersed in a thermostated glycerine bath as shown in Fig. 2, with the junction of the reference thermo-couple inserted in the bath, thus serving as reference system.

The bath also contained heaters, cooling coil, thermostat thermometer, and stirrer, not shown in Fig. 2. The temperature difference between bath and autoclave was registered by a recorder (Philips PR2210 A/21).

Apparatus for chemical analysis experiments. In this case the reaction was performed in small glass autoclaves (about $25 \mathrm{ml}$ ) manufactured from pilot plant pipes (5/8") with spherical joints. They were sealed by melting at one end and the spherical joint clamped with loose flanges.

Acta Chem. Scand. 20 (1966) No. 2 


\section{Performance of experiments}

Thermographic analysis. The aluminium trichloride was sieved in five fractions with the following limits of particle size $>950,950-500,500-250,250-100,<100 \mu \mathrm{m}$, and with the weight distribution $5,20,30,30,15 \%$, respectively, in round numbers. When the first fraction had been discarded, each was used for experiments at 40, 50, and $60^{\circ} \mathrm{C}$ except for methyl chloride where only fractions 2 and 5 were used.

The autoclave was charged with $10.0 \mathrm{~g}$ aluminium trichloride sealed in a glass ampoule, $10.5 \mathrm{~g}$ phosphorus trichloride, and alkyl chloride in about $100 \%$ excess. To facilitate

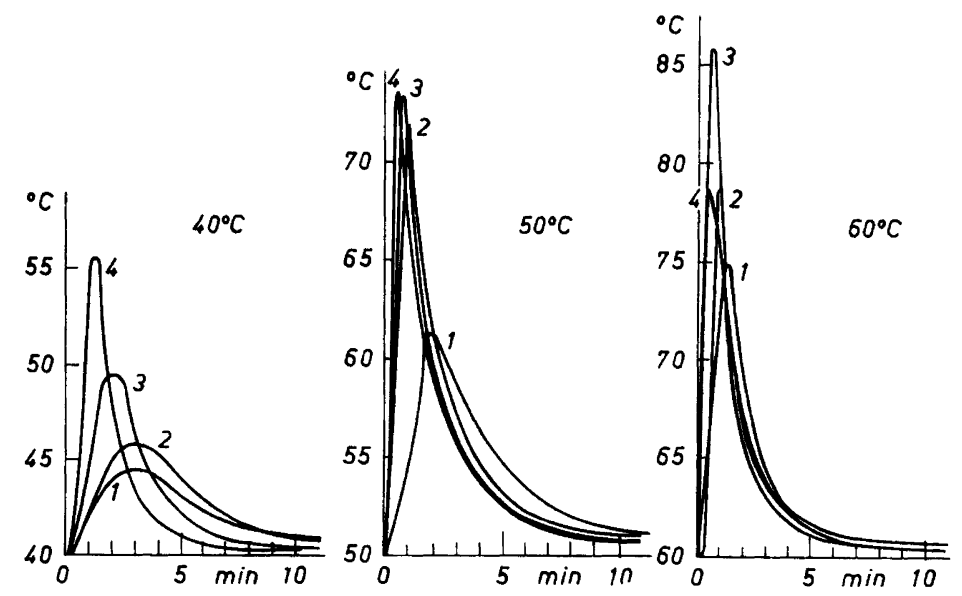

Fig. 3. Temperature-time curves for the ethyl-complex synthesis at 40,50 , and $60^{\circ} \mathrm{C}$. The limits of particle size of the aluminium chloride $1,500-950 \mu \mathrm{m} ; 2,250-500 \mu \mathrm{m}$; $3,100-250 \mu \mathrm{m} ; 4,<100 \mu \mathrm{m}$.

the addition of the low boiling methyl and ethyl chloride, the autoclave was first cooled with ice water or dry ice and alcohol.

With the autoclave arranged as shown in Fig. 2, the reaction was started when temperature equilibrium was reached, by starting the stirrer, which immediately crushed the

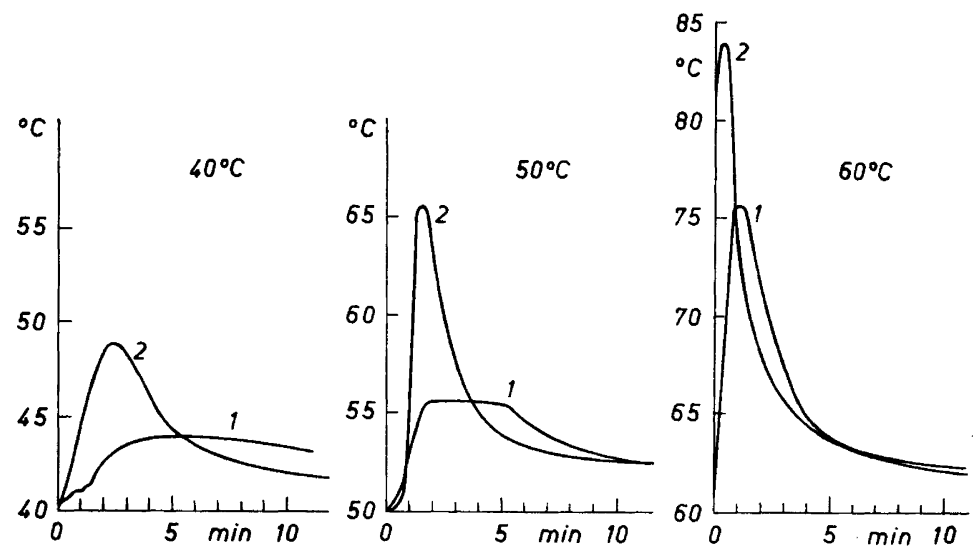

Fig. 4. Temperature-time curves for the methyl-complex synthesis at 40,50 , and $60^{\circ} \mathrm{C}$. The limits of particle size of the aluminium chloride $1,500-950 \mu \mathrm{m} ; 2,<100 \mu \mathrm{m}$.

Acta Chem. Scand. 20 (1966) No. 2 
Fig. 5. Distribution of $\mathrm{PCl}_{3}(\times)$, $\mathrm{C}_{2} \mathrm{H}_{5} \mathrm{PCl}_{3}+\mathrm{Al}_{2} \mathrm{Cl}_{7}^{-}(0)$ and $\mathrm{C}_{2} \mathrm{H}_{6} \mathrm{PCl}_{3}+\mathrm{AlCl}_{4}^{-}$

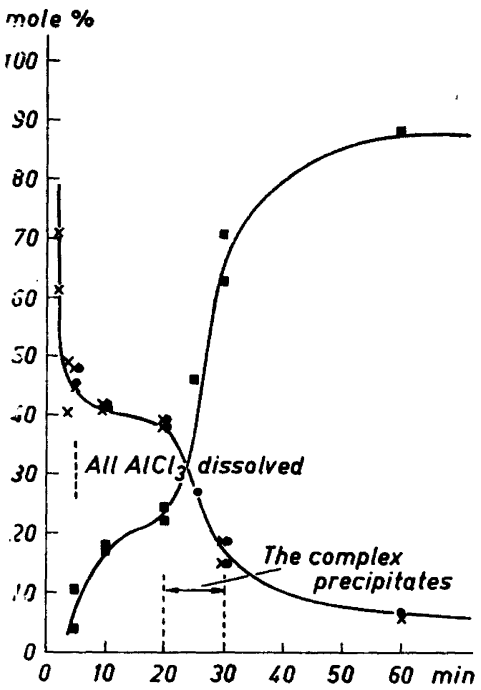

glass ampoule with aluminium trichloride. On the recorder the curves shown in Figs. 3 and 4 were obtained.

Estimation of phosphorus trichloride concentration. The small glass autoclaves were charged with stoichiometric quantities of aluminium trichloride and phosphorus trichloride (each in the range 3-4 g) and then cooled with ice water before the ethyl chloride in excess was added. No reaction takes place at $0-5^{\circ} \mathrm{C}$. The autoclave was immersed in a water bath thermostated at $50^{\circ} \mathrm{C}$. The time was measured from that moment until the autoclave was transferred to another bath with ice water thus terminating the reaction. The small glass autoclave was screwed apart, and the halves carefully placed in a larger glass autoclave manufactured from 2" pilot plant pipes and containing some ice water. When this was rotated, the water flowed down into the small autoclave and hydrolysed the reaction mixture. After a while the solution had cooled and could be poured into a measuring flask for analysis. The result is given in a diagram in Fig. 5.

Analytical method. Phosphate can be estimated colorimetrically with ammonium molybdate and vanadate as a color developer. ${ }^{\circ}$ With strong oxidizing agents as nitric acid and perchloric acid in the presence of molybdate as catalyst, all phosphorus compounds are transferred to phosphate. With a mild oxidizing agent such as potassium permanganate, only phosphorus trichloride is oxidized and can be estimated in the presence of carbon bound phosphorus, which does not affect the measurements.

\section{RESULTS}

The primary results of the thermographic analysis are demonstrated in Figs. 3 and 4. The general shape of the obtained curves demonstrates one first stage (Stage I) with vivid heat evolution and a second stage (Stage II) where heat is evolved comparatively slowly. The later stage is described in the graph as a curve nearly parallel to and very near the time-axis but is not shown completely in the figures because of the long time (several hours) to reach the base line. Moreover, the overall reaction velocity in the first stage depends on particle size of the aluminium trichloride and is higher with smaller particles. It was also demonstrated in a separate experiment that $100^{\circ} \mathrm{C}$ gives

Acta Chem. Scand. 20 (1966) No. 2 
a reasonable reaction velocity for preparative purpose in stage II. Especially ethyl chloride gives problems due to low reaction velocity in that stage.

The results of the phosphorus trichloride consumption experiments with ethyl chloride are demonstrated in Fig. 5 . It is evident that when about $50 \%$ of the phosphorus trichloride has reacted, which happens relatively rapidly, all aluminium trichloride has been consumed.

This indicates that stage $\mathrm{I}$, as it appears also from thermographic analysis, ends by the complex $\mathrm{C}_{2} \mathrm{H}_{5} \mathrm{PCl}_{3}+\mathrm{Al}_{2} \mathrm{Cl}_{7}-$. This complex has been described by Hoffmann, ${ }^{10}$ who has also suggested it as an intermediate in the reaction. The slower stage II leeds to practically complete consumption of phosphorus trichloride. The melting points for $\mathrm{CH}_{3} \mathrm{PCl}_{3}{ }^{+} \mathrm{Al}_{2} \mathrm{Cl}_{7}^{-}$and $\mathrm{CH}_{3} \mathrm{PCl}_{3}+\mathrm{AlCl}_{4}^{-}$in a pure state are $41^{\circ} \mathrm{C}$ and $280-285^{\circ} \mathrm{C}$, respectively. In the last case, the melting is accompanied by a slow decomposition, therefore the uncertainty in estimation in spite of reapeated recrystallization in methylene chloride and careful drying in dry nitrogen. The melting point for $\mathrm{C}_{2} \mathrm{H}_{5} \mathrm{PCl}_{3}+\mathrm{AlCl}_{4}{ }^{-}$prepared by pure reagents lies in the same temperature range. As a rule much lower melting points are found due to high sensitivity to impurities.

\section{DISCUSSION}

Both Clay $^{7}$ and Kinnear-Perren ${ }^{8}$ have proposed mechanisms for the synthesis of the complexes. However, a third mechanism suggested by Cade ${ }^{12}$ 1958 has been more generally accepted. This theory involves a Friedel-Crafts mechanism with a carbonium cation that is added to the free electron-pair of phosphorus in phosphorus trichloride and can be summed up as follows.

$$
\begin{aligned}
& \mathrm{RCl}+\mathrm{AlCl}_{3} \rightleftharpoons \mathrm{R}^{+} \mathrm{AlCl}_{4}^{-} \rightleftharpoons \mathrm{R}^{+}+\mathrm{AlCl}_{4}^{-} \\
& \mathrm{R}^{+}+\mathrm{PCl}_{3} \longrightarrow \mathrm{RPCl}_{3}^{+} \\
& \mathrm{RPCl}_{3}^{+}+\mathrm{AlCl}_{4}^{-} \longrightarrow \mathrm{RPCl}_{3}+\mathrm{AlCl}_{4}^{-}
\end{aligned}
$$

The mechanism seems very probable because it can explain why higher alkyl chlorides always are isomerised. ${ }^{8}$ However, some questions can be presented, which will not be explained by this mechanism. One expects, for instance, to find close analogies between the akylation of phosphorus trichloride and the condensation of alkyl chloride and benzene in a FriedelCrafts reaction. This is not the case, however, since the Clay-Kinnear-Perren condensation always leads to an isomerisation to $100 \%$ of higher alkyl groups ${ }^{8}$ and has the serious disadvantage that carbon chains with more than four carbon atoms are broken down, and a product which mainly contains tertbutyl groups is obtained. ${ }^{13}$

A Friedel-Crafts condensation as a rule leads to a mixture of isomerised and unisomerised products and a shortening of the carbon chain is very unusual.

Thus, as it is quite clear that the mechanism has to involve free carbonium cations, it is difficult to see how methyl chloride can take part in a reaction with phosphorus trichloride and aluminium trichloride with the high velocity the thermographic measurements has shown, although it is known that practically no $\mathrm{CH}_{3}{ }^{+}$-ions are formed in methyl chloride in the presence of aluminium trichloride. 
Because of these questions, another mechanism seems more probable, and due to the direction of the dipole moment of phosphorus trichloride, the following reaction is proposed

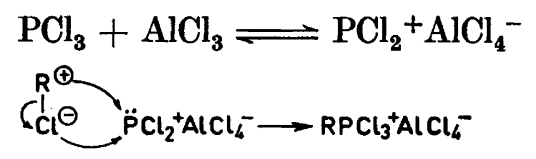

The electron pair deficit in aluminium trichloride is filled up by a chlorine atom from phosphorus trichloride. The $\mathrm{PCl}_{2}{ }^{+}$-ion thus formed attacks the chlorine atom in alkyl chloride and breaks the chlorine carbon bond. $A$ free carbonium cation thus is generated before it adds to the free electron pair of phosphorus. This mechanism which implies an elucidation of a theory outlined. by Kinnear and Perren also fully explains the isomerization of the alkyl group.

A complex ${ }^{14}$ between phosphorus trichloride and aluminium trichloride has never been indicated but as an argument for the existence of an unstable complex, it can be stated that such a complex ${ }^{15}$ exists between phosphorus trichloride and gallium trichloride which also readily condenses with alkyl chloride.

As further proof of the existence of an unstable complex, two reactions can be mentioned which can only be explained by an equilibrium

$$
\mathrm{AlCl}_{3}+\mathrm{PCl}_{3} \rightleftharpoons \mathrm{PCl}_{2}+\mathrm{AlCl}_{4}^{-}
$$

The first implies the well-known substitution of hydrogen in benzene with a $\mathrm{PCl}_{2}$-group in a reaction with phosphorus trichloride in the presence of aluminium trichloride. The other reaction concerns addition of phosphorus trichloride to ethylene in the presence of aluminium trichloride which leads to the product $\mathrm{ClCH}_{2}-\mathrm{CH}_{2} \mathrm{PCl}_{2} \cdot{ }^{16}$

The proposed mechanism can be extended to explain the reactions with partially substituted phosphorus trichloride which in these cases starts with aluminium trichloride and the phosphorus compound forming a stable complex. As substituents alkyl and aryl groups ${ }^{8,17-20}$ are concerned.

With the mechanism here suggested, stage I may thus be described by the following reaction sequence.

$$
\begin{aligned}
& \mathrm{AlCl}_{3}+\mathrm{PCl}_{3} \rightleftharpoons \mathrm{PCl}_{2}+\mathrm{AlCl}_{4}^{-} \\
& \mathrm{PCl}_{2}+\mathrm{AlCl}_{4}^{-}+\mathrm{RCl} \longrightarrow \mathrm{RPCl}_{3}+\mathrm{AlCl}_{4}^{-} \\
& \mathrm{RPCl}_{3}+\mathrm{AlCl}_{4}^{-}+\mathrm{AlCl}_{3} \longrightarrow \mathrm{RPCl}_{3}+\mathrm{Al}_{2} \mathrm{Cl}_{7}^{-}
\end{aligned}
$$

where reaction (1), as demonstrated by the dependence of reaction velocity on particle size of aluminium chloride, has a considerable influence on the total reaction velocity. Stage II which, in the temperature interval $40-100^{\circ} \mathrm{C}$, is slower than $\mathrm{I}$ leads to the complex $\mathrm{RPCl}_{3}{ }^{+} \mathrm{AlCl}_{4}^{-}$presumably as follows

$$
\begin{aligned}
& \mathrm{RPCl}_{3}+\mathrm{Al}_{2} \mathrm{Cl}_{7}^{-} \rightleftharpoons \mathrm{RPCl}_{3}+\mathrm{AlCl}_{4}^{-}+\mathrm{AlCl}_{3} \\
& \mathrm{AlCl}_{3}+\mathrm{PCl}_{3} \rightleftharpoons \mathrm{PCl}_{2}+\mathrm{AlCl}_{4}^{-} \\
& \mathrm{PCl}_{2}+\mathrm{AlCl}_{4}^{-}+\mathrm{RCl} \rightarrow \mathrm{RPCl}_{3}+\mathrm{AlCl}_{4}^{-}
\end{aligned}
$$


In stage $I$ it was demonstrated that reactions (5) and (6), there nominated (1) and (2), are relatively rapid reactions. Thus (4) has to be rate determining in the over all reaction in stage II and also for the total process (stages I + II).

It is most likely that the dissociation inherent in (4) $\mathrm{Al}_{2} \mathrm{Cl}_{7}^{-} \rightleftharpoons \mathrm{AlCl}_{4}^{-}+$ $\mathrm{AlCl}_{3}$ is rate determining. For preparative purpose, a temperature of about $100^{\circ} \mathrm{C}$ as reaction temperature in preparing the methyl or ethyl complex seems resonable with regard to the observed reaction time (about $30 \mathrm{~min}$ ). Since stage I can be performed relatively fast within broad limits of particle size of the aluminium trichloride, special sieving of the commercial aluminium trichloride should in most cases not be necessary.

\section{REFERENCES}

1. Kosolapoff, G. M. Organophosphorus Compounds, John Wiley and Sons, New York 1950, Chap. 7, p. 121.

2. Crofts, P. C. Quart. Rev. 12 (1958) 341.

3. Frank, A. W. Chem. Rev. 461 (1961) 389

4. Jungerman, E. and McBride, J. J. J. Org. Chem. 26 (1961) 4148.

5. Maier, L. Angew. Chem. 71 (1959) 574.

6. Angstadt, H. P. J. Am. Chem. Soc. 86 (1964) 5040.

7. Clay, J. P. J. Org. Chem. 16 (1951) 892.

8. Kinnear, A. M. and Perren, E. A. J. Chem. Soc. 19523437.

9. Quinland, K. P. and DeSessa, M. A. Anal. Chem. 27 (1955) 1626.

10. Hoffmann, F. W., Simmons, T. C. and Glunz, L. J. J. Am. Chem. Soc. 79 (1957) 3570.

11. White, N. R., Scott, L. B., Kister, A. T., Redlich, O. and Miller, R. W. Final Report on Contract No. W 18-108-CM-1322, Report N. S.-13238, Sept. 30, 1950.

12. Cade, J. A. Tetrahedron 2 (1958) 322.

13. Metzger, S. H., Basedow, O. H. and Isbell, A. F. J. Org. Chem. 29 (1964) 627.

14. Holmes, R. R. J. Inorg. Nucl. Chem. 12 (1960) 266.

15. Greenwood, N. N., Perkins, P. G. J. Chem. Soc. 1957 4345; Pure Appl. Chem. 2 (1961) 55.

16. Titov, A. I., Sizova, M. V. and Gitel, P. O. Dokl. Akad. Nauk. USSR 159 (1964) 385.

17. Ferron, J. L. Can. J. Chem. 39 (1961) 842.

18. Ivin, S. Z. and Karavanov, K. V. Zh. Obshch. Khim. 28 (1958) 2958.

19. Biddle, P., Kennedy, J. and Williams, J. L. Chem. Ind. (London) 1957 (1481.

20. Ivin, S. Z., Karavanov, K. V. and Lysenko, V. V. Zh. Obshch. Khim. 34 (1964) 852.

Received November 4, 1965. 